\title{
Bispectral index and their relation with consciousness of the patients who receive desflurane or sevoflurane anesthesia during wake-up test for spinal surgery for correction
}

\author{
Tae Kyoung Seol, Min Kyu Han, Hee Jong Lee, Mi Ae Cheong, and Jong Hun Jun \\ Department of Anesthesiology and Pain Medicine, Hanyang University College of Medicine, Seoul, Korea
}

Background: Wake-up tests may be necessary during surgery for kypho-scoliosis to ensure that spinal function remains intact. It is difficult to predict the time when patients can respond to a verbal command. We evaluated the effectiveness of the bispectral index (BIS) and its relation to patients' levels of consciousness in wake-up tests during desflurane and sevoflurane anesthesia.

Methods: Eighteen patients each were enrolled in the desflurane and sevoflurane groups for spinal correction surgery. We measured BIS values, blood pressure, heart rate, and consciousness state and time, at the points when patients responded during the wake-up test.

Results: The BIS values when patients made fists upon a verbal command (T3) were $86.7 \pm 7.5$ for desflurane and $90.3 \pm 5.4$ for sevoflurane. Patients in the desflurane group had significantly shorter wake up delays than those in the sevoflurane group $(6.9 \pm 1.8 \mathrm{~min}$ vs. $11.8 \pm 3.6 \mathrm{~min})$. However, there was no difference between the groups in the time between the response to a verbal command and the time when a patient moved their toes in response to verbal commands. No recall of the wake-up tests occurred in either group.

Conclusions: The values obtained using the BIS index could to some extent predict the time of a patient's and would be informative during desflurane and sevoflurane anesthesia. Moreover, desflurane permitted faster responses to verbal commands than sevoflurane, and allowed the wake-up test to be performed sooner. (Korean J Anesthesiol 2012; 62: 13-18)

Key Words: Bispectral index, Spinal surgery, Wake-up test.

Received: April 6, 2011. Revised: 1st, July 18, 2011; 2nd, August 1, 2011. Accepted: August 1, 2011.

Corresponding author: Jong Hun Jun, M.D., Department of Anesthesiology and Pain Medicine, Hanyang University Hospital, Haengdang-dong, Sungdong-gu, Seoul 133-792, Korea. Tel: 82-2-2290-8680, Fax: 82-2-2299-8692, E-mail: jhjun@hanyang.ac.kr

(c) This is an open-access article distributed under the terms of the Creative Commons Attribution Non-Commercial License (http:// creativecommons.org/licenses/by-nc/3.0/), which permits unrestricted non-commercial use, distribution, and reproduction in any medium, provided the original work is properly cited. 


\section{Introduction}

Decompression and spinal fusion are surgical treatments widely used for spinal lesions due to scoliosis and ankylosing spondylitis. Because these surgical reductions can cause neurological side-effects including damage to the spinal cord, studies have investigated methods to detect and prevent such side effects. The current widely used methods of prevention are electrophysiological monitoring (somatosensory evoked potentials, spinal evoked potentials, and motor evoked potentials) and clinical monitoring (the wake-up test) [1-4]. The wake-up test is a simple, economical, and relatively accurate procedure that is useful because it can monitor perioperative spinal cord damage.

The bispectral index (BIS) is an indicator of the effects of anesthetics and sedatives on sleep and level of consciousness. There have been many investigations of the relationship between BIS index and patient awakening during anesthesia. However there have been few studies concerning the relationship between BIS index and the wake-up test.

Among the anesthesias that require perioperative wakeup tests are intravenous (IV) anesthesia (using fentanyl with or without nitrous oxide) and balanced anesthesias which have a fast emergence from anesthesia. However, there is a greater possibility of patient recall during IV anesthesia than during inhalation anesthesia. Desflurane and sevoflurane have relatively fast onset times and awakening times, and are appropriate for use in surgical procedures that require such tests $[5,6]$. However, it is very difficult to predict the response times of patients in such tests because they depend on the inhalational anesthetic used. Therefore, if a patient's state of consciousness can be established during the test by using the BIS, this information can be helpful in predicting the appropriate times for giving verbal commands to the patient. Therefore, we measured BIS during surgery under anesthesia with sevoflurane and desflurane, which have relatively short awakening times, and examined these relationships.

\section{Materials and Methods}

After obtaining approval from our Institutional Review Board and written informed consent from the patients, 18 patients of American Society of Anesthesiologists (ASA) class II or below who were to undergo spinal fusion for scoliosis or ankylosing spondylitis were chosen for the study. Patients with neurological disorders, hearing disorders or epilepsy, and patients who had taken anticonvulsants, sedatives, and stimulants were excluded. The day before surgery, the wake-up test was explained and the patients were told that the examiner would ask them to make a fist and wiggle their toes during the test. The day after surgery, the patients were asked if they could recall the examiner's commands.

All operations were performed by the same orthopedic surgeon. No premedication was given. Basic monitoring devices, such as a noninvasive blood pressure monitor, EKG, and pulse-oximeter, and a respiratory circuit to monitor $\mathrm{CO}_{2}$, were observed throughout the surgery.

To measure BIS, a disposable BIS Sensor connected to the BIS Model A-2000 monitor (Aspect Medical Systems, USA) was attached to the patient's forehead before inducing anesthesia. BIS was measured during the entire period of anesthesia in all patients. To induce anesthesia, thiopental sodium (200-250 mg) and rocuronium (50 mg) or vecuronium ( $8 \mathrm{mg}$ ) were intravenously administered and the patients were intubated. Patients were randomly divided into 2 groups of 9 individuals receiving either sevoflurane or desflurane as a general anesthetic, combined with oxygen and nitrous oxide. One patient in the sevoflurane group was excluded due to malfunction of the BIS monitor. End-tidal concentration in the sevoflurane group was maintained at $1-3$ vol\%, and in the desflurane group at 4-8 vol\%. During surgery, both groups were maintained at 1-2 MAC. Oxygen was administered at a flow rate of $1 \mathrm{~L} / \mathrm{min}$, and nitrous oxide was administered at $2 \mathrm{~L} / \mathrm{min}$. Blood pressure and central venous pressure were measured by cannulation of the radial artery and internal jugular vein, respectively. Body temperature was maintained at 36 degrees with an air warmer. For adequate muscle relaxation, a train of four was frequently applied with a peripheral nerve stimulator (TOF-Watch, Organon Teknika, The Netherlands). When 4 twitches were observed, additional rocuronium (10 $\mathrm{mg}$ ) or vecuronium ( $2 \mathrm{mg}$ ) was administered. The patient was placed in the prone position for surgery. Mechanical ventilation was performed and $\mathrm{PaCO}_{2}$ remained between 35 and $40 \mathrm{mmHg}$. Apart from during the wake-up test, deliberate hypotension was produced to maintain the mean blood pressure between $50-65 \mathrm{mmHg}$ using sodium nitroprusside $(0.3-3 \mu \mathrm{g} / \mathrm{kg} /$ $\mathrm{min}$ ) to reduce blood loss. Hematocrit was maintained at $20-$ $30 \%$, and packed red blood cells (PRBC) were administered when needed. The surgeon corrected the spinal deformity, and after immobilizing the apparatus, when the wake-up test was requested, the administration of inhalational anesthetics was stopped, and only oxygen was administered. During the wakeup test, fentanyl $(1.5 \mu \mathrm{g} / \mathrm{kg})$ for pain-relief, and neostigmine (2 mg) and glycopyrrolate ( $0.4 \mathrm{mg}$ ) for reversal of muscle relaxation were intravenously administered. The administration of sodium nitroprusside for deliberate hypotension was stopped. After confirming full reversal of the muscle relaxants with a peripheral nerve stimulator, the anesthesiologist held the patient's hand and requested the patient to make a fist at 30 second intervals to check the level of awareness. If the patient 
could make a very tight fist, it was considered that he had recovered consciousness. He was then was asked to move his toes. If he was able to do so, the wake-up test was pronounced negative and it was deemed that there was no damage to the spinal cord. Thiopental sodium (200-250 mg) and rocuronium (50 mg) or vecuronium (8 mg) were immediately administered, and inhalational anesthetics and nitrous oxide were readministered. The anesthesia was maintained until the end of the surgery. The entire procedure of the wake-up test was performed by a single anesthesiologist.

The time at the beginning of surgery was recorded as T0. The point when the administration of inhalational anesthetics was stopped for the wake-up test was recorded as $\mathrm{T} 1$. T2 was when the BIS value was 85 . The time when the patient responded to a verbal command and made a fist was T3, and when he moved his toes on command was T4. T5 was 1 minute after the readministration of inhalational anesthetics which followed the wake-up test and IV administration of thiopental sodium and muscle relaxants. Each time point, as well as mean BP, heart rate, and BIS values at these points, was recorded.

In the ward, fentanyl and ketorolac tromethamine were administered (iv) with patient-controlled analgesia (PCA). The next day, the patient was asked if he had heard the verbal commands and felt pain during the wake-up test.

The data were analyzed using Sigmastat software (version 2.03). The data concerning the surface roughness were not

Table 1. Demographic Data

\begin{tabular}{lcc}
\hline & Desflurane & Sevoflurane \\
\hline Sex (M/F) & $2: 7$ & $3: 5$ \\
Age (yr) & $18.2 \pm 11.9(15,13-50)$ & $17.3 \pm 9.4(14,11-40)$ \\
Height $(\mathrm{cm})$ & $152.6 \pm 12.5(145,143-171)$ & $149.4 \pm 10.7(145.5,144-169)$ \\
Weight $(\mathrm{kg})$ & $45.8 \pm 7.2(45,39-63)$ & $43.6 \pm 5.7(42.5,38-58)$ \\
Anesthesia duration (min) & $482 \pm 85(461,390-621)$ & $502 \pm 110(491.5,364-650)$ \\
\hline
\end{tabular}

Values are means \pm SD (median, range). There were no significant differences between the groups.
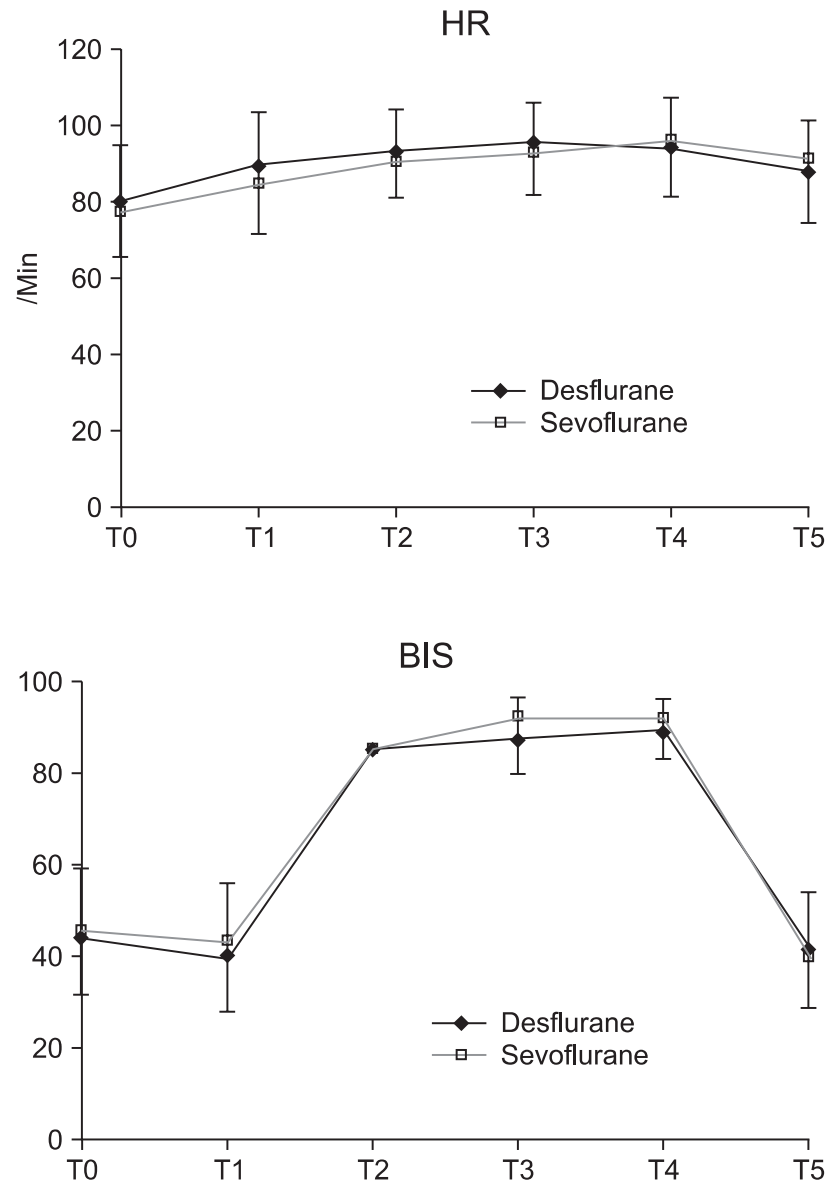

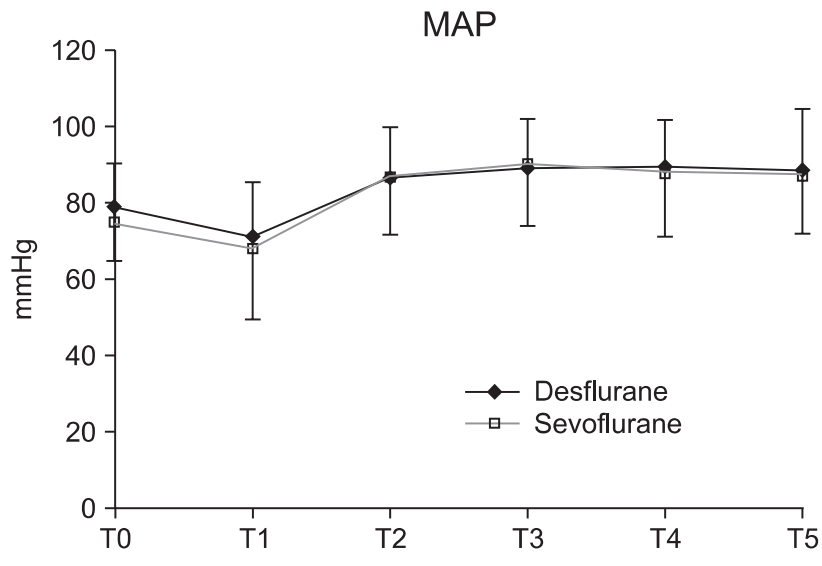

Fig. 1. Changes in heart rate, mean arterial pressure and bispectral index during the wake-up test. There were no significant differences between the groups. T0: Start of surgery, T1: Time of discontinuation of anesthetics for the wake-up test, T2: Time of BIS value of 85, T3: Time of patient's response to verbal command, T4: Time of patient's moving toes in a response to verbal command, T5: 1 minute after re-administration of anesthetics, BIS: bispectral index, MAP: mean arterial pressure, HR: heart rate. 
normally distributed (Kolmogorov-Smirnov test). Therefore, all levels were compared using nonparametrical analysis. The measured time points, BIS values, mean BP rates, and heart rates of the 2 groups were compared using the Mann-Whitney test. $\mathrm{P}<0.05$ (two-sided test) was considered significant.

\section{Results}

The 2 groups did not differ with respect to demographic data (Table 1). At the point when the patient made a fist upon a verbal command (T3), BIS values were $86.7 \pm 7.5$ for desflurane and $90.3 \pm 5.4$ for sevoflurane. At the point when the patient was told to move his toes (T4), the BIS values were 88.7 for desflurane \pm 6.1 and $91.4 \pm 4.9$ for sevoflurane. There were no significant differences between the 2 groups with regard to any time point. There were no significant differences in mean BP and heart rates at any point in time (Fig. 1).

The time taken for the BIS value to reach 85 after the administration of inhalational anesthetics was stopped (T2-T1) was $6.4 \pm 1.9 \mathrm{~min}$ for the desflurane group and $11.5 \pm 3.8 \mathrm{~min}$ for sevoflurane group. The interval from the point when administration of inhalational anesthetics was stopped to the time of response to a verbal command (T3-T1) for the desflurane group was $6.9 \pm 1.8 \mathrm{~min}$ and $11.8 \pm 3.6 \mathrm{~min}$. for the sevoflurane group. The intervals $\mathrm{T} 2-\mathrm{T} 1$ and $\mathrm{T} 3-\mathrm{T} 1$ were significantly different in the 2 groups $(\mathrm{P}<0.05)$ (Table 2$)$. All wake-up test results were negative. No damage occurred to any spinal cord after the immobilization device had been secured. Also, the day after surgery, all patients responded negatively to the question on whether they recalled the perioperative wake-up test.

\section{Discussion}

Damage caused by spinal surgery can be clinically monitored by the wake-up test [7-9] and ankle clonus test [2]. The wake-up test requires no additional equipment or personnel and is economical and easy to use [10]. Although it can be accompanied by extubation accidents from excessive stimulation, coughing, trauma from excessive movement, failure of the immobilization device, mental disorder from perioperative memories, air embolism, or myocardial ischemia, these side-effects can be minimized by proper explanation, appropriate drug choice and usage, and careful anesthesia $[2,11]$. However, the wake-up test has the highest sensitivity and specificity, and is most often used $[5,6,12]$.

The appropriate BIS value requiring the minimum extent of sedation in the Department of Radiology is $80-85$. At this value and beyond, orientation reportedly returns in most cases $[13,14]$. According to past reports [5,6,12], the average BIS value which made it possible to perform a wake-up test was 90 . Our data suggest that the wake-up test should be performed at a BIS value of 85 .

Desflurane and sevoflurane have relatively low solubilities and blood/gas partition coefficients in blood and tissues (desflurane 0.45, sevoflurane 0.65). Hence onset times and awakening times are very fast. The wake-up tests in the present study could be performed in a relatively short time after perioperative administration of the anesthetics was stopped. The patients under desflurane responded earlier to the verbal command than those under sevoflurane, and their wake-up tests could be performed sooner. The fast recovery is assumed to be due to the low solubility of desflurane compared to sevoflurane. This is very important for wake up tests because patients need rapid awakening, and this represents a major advantage of desflurane [15]. McCann et al. [5] reported that after stopping the administration of anesthetics, the reaction time to the verbal command in the wake-up test for isoflurane was $8.9 \pm 4.9 \mathrm{~min}$ and $8.9 \pm 2.1 \mathrm{~min}$ for fentanyl. Ting et al. [6] stated that for desflurane it was $4.1 \pm 0.6 \mathrm{~min}$ and for fentanyl 8.9 $\pm 2.1 \mathrm{~min}$. In the present study it was $6.9 \pm 1.8 \mathrm{~min}$ for desflurane and $11.8 \pm 3.6 \mathrm{~min}$ for sevoflurane. The measurements from different studies may differ depending on the method of anesthesia used, but taking all the results into account, it appears that the wake-up test can be performed sooner with desflurane.

In the wake-up test, awakening from anesthesia requires the patient not only to respond to the verbal command but also to regain control of his body. Because of this, the recovery time from anesthesia can differ depending on the type of anesthetic used. With desflurane and sevoflurane, there was no difference

Table 2. Time Variables during the Wake-up Test

\begin{tabular}{|c|c|c|c|}
\hline & Desflurane (n: 9) & Sevoflurane (n:8) & $P$ value \\
\hline T1-T0 (min) & $226 \pm 73(238,123-331)$ & $242 \pm 86(272,116-359)$ & NS \\
\hline $\mathrm{T} 2-\mathrm{T} 1$ (min) & $6.4 \pm 1.9(6.5,3.8-9.4)$ & $11.5 \pm 3.8(11.1,6.3-16.2)$ & 0.034 \\
\hline T3-T2 (min) & $0.5 \pm 0.3(0.4,0.2-1.2)$ & $0.7 \pm 0.5(0.5,0.3-1.9)$ & NS \\
\hline T3-T1 (min) & $6.9 \pm 1.8(5.9,4.9-9.7)$ & $11.8 \pm 3.6(11.45,7.3-17.1)$ & 0.020 \\
\hline T4-T3 (min) & $0.2 \pm 0.1(0.2,0.1-0.35)$ & $0.3 \pm 0.2(0.31,0.08-0.62)$ & NS \\
\hline
\end{tabular}

Values are mean \pm SD (median, range). T0: Start of surgery, T1: Time of discontinuation of anesthetics for wake-up test, T2: Time of BIS value of 85, T3: Time of patient's response to verbal command, T4: Time of patient's moving toes in response to verbal command, NS: no significance. 
in the time required for patients to awake from anesthesia, but the time needed for patients to recollect their names and dates of birth were significantly shorter for desflurane In the short orientation memory concentration test, there was a 15-minute difference after extubation, but no subsequent difference $[16,17]$. The slow orientation response can be understood as the latency period between the time the patient responds to the verbal command and the time he responds to the command to move his toes in the present investigation. Anesthesia with fentanyl increased the latency period in this study, and this latency period can be considered to correspond to T4-T3, which did not differ between desflurane and sevoflurane. These 2 inhalational anesthetics have similar and relatively fast awakening times. There is a need for future studies of the latency periods of inhalational and IV anesthetics with long awakening times.

In the wake-up test, the BIS values at the time patients responded to the verbal commands and recovered consciousness were $86.7 \pm 7.5$ in the desflurane group and $90.3 \pm 5.4$, in the sevoflurane group; which were similar to the values reported by researchers using other anesthetics. These values are significant in that that can predict, to a certain extent, the time a patient will respond. In the present study using a perioperative wake-up test, if the BIS value increased rapidly it meant that the patient had recovered consciousness. Such rapid increases are in line with past reports. For hypotensive anesthesia, the drugs used on blood vessels can affect the autonomic nervous system when the patient is awakened from anesthesia, Therefore, rather than predicting awakening based on heart rate and blood pressure, the BIS index has proved to be helpful for monitoring the level of consciousness.

The sensitivity and specificity of the BIS value can differ greatly depending on the anesthetic method used and the patient, but it is noteworthy that there is a trend towards using monitoring devices such as wake-up tests in which the possibility of a patient recalling events during anesthesia is high. None of the patients in the present study recalled anything about the perioperative wake-up test. This may be because the inhalational anesthetics used induce stronger memory loss, and anesthetized the patient for longer periods of time than opioid IV anesthetics. Future research should investigate multiple situations with large numbers of test patients using a variety of anesthetics and methods of anesthesia in order to establish when the wake-up test can be performed. Also, we need to determine whether recall of the perioperative wake-up test can be monitored by BIS.

There are some limitations in this study. The sample size was small, and a larger sample size study might reveal a different result. The other limitation is that BIS values might not reliably differentiate consciousness from unconsciousness, because
BIS varies in sensitivity and specificity between anesthetic techniques and individual patients [18]. However, BIS may provide useful trend information for any given patient and it has been widely used during sedation and anesthesia. In addition, BIS values do not appear to be independent of the anesthetic agent administered, therefore comparable BIS values achieved with different agents (inhalational vs IV) may not represent the same depth of anesthesia. However, there would not be much difference in our study because we used inhalational anesthetics.

In conclusion, the values obtained using the BIS index showed that they can, to some extent, predict the time of a patient's response and such information would be informative during desflurane and sevoflurane anesthesia. Moreover, desflurane permitted faster responses to verbal commands than sevoflurane, and thus allowed the wake-up test to be performed sooner.

\section{References}

1. Vauzelle C, Stagnara P, Jouvinroux P. Functional monitoring of spinal cord activity during spinal surgery. Clin Orthop Relat Res 1973; (93): 173-8.

2. Hall JE, Levine CR, Sudhir KG. Intraoperative awakening to monitor spinal cord function during Harrington instrumentation and spine fusion. Description of procedure and report of three cases. J Bone Joint Surg Am 1978; 60: 533-6.

3. Pathak KS, Brown RH, Nash CL Jr, Cascorbi HF. Continuous opioid infusion for scoliosis fusion surgery. Anesth Analg 1983; 62: 841-5.

4. Ben-David B. Spinal cord monitoring. Orthop Clin North Am 1988; 19: 427-48.

5. McCann ME, Brustowicz RM, Bacsik J, Sullivan L, Auble SG, Laussen PC. The bispectral index and explicit recall during the intraoperative wake-up test for scoliosis surgery. Anesth Analg 2002; 94: 1474-8.

6. Ting CK, Hu JS, Teng YH, Chang YY, Tsou MY, Tsai SK. Desflurane accelerates patient response during the wake-up test for scoliosis surgery. Can J Anaesth 2004; 51: 393-7.

7. Crawford AH, Jones CW, Perisho JA, Herring JA. Hypnosis for monitoring intraoperative spinal cord function. Anesth Analg 1976; 55: 42-4.

8. Waldman J, Kaufer H, Hensinger RN, Callaghan ML, Lieding KG. Wake-up technic to avoid neurologic sequelae during Harrington rod procedure: a case report. Anesth Analg 1977; 56: 733-5.

9. Lueders H, Gurd A, Hahn J, Andrish J, Weiker G, Klem G. A new technique for intraoperative monitoring of spinal cord function: multichannel recording of spinal cord and subcortical evoked potentials. Spine (Phila Pa 1976) 1982; 7: 110-5.

10. Dorgan JC, Abbott TR, Bentley G. Intra-operative awakening to monitor spinal cord function during scoliosis surgery. Description of the technique and report of four cases. J Bone Joint Surg Br 1984; 66: 716-9.

11. Abott TR, Bentley G. Intra-operative awakening during scoliosis surgery. Anaesthesia 1980; 35: 298-302. 
12. Blussé van Oud-Alblas HJ, Peters JW, de Leeuw TG, Vermeylen KT, de Klerk LW, Tibboel D, et al. A comparison in adolescents of composite auditory evoked potential index and bispectral index during propofol-remifentanil anesthesia for scoliosis surgery with intraoperative wake-up test. Anesth Analg 2008; 107: 1683-8.

13. Bell JK, Laasch HU, Wilbraham L, England RE, Morris JA, Martin DF. Bispectral index monitoring for conscious sedation in intervention: better, safer, faster. Clin Radiol 2004; 59: 1106-13.

14. Dahaba AA, Lischnig U, Kronthaler R, Bornemann H, Georgiev V, Rehak PH, et al. Bispectral-index-guided versus clinically guided remifentanil/propofol analgesia/sedation for interventional radiological procedures: an observer-blinded randomized study. Anesth Analg 2006; 103: 378-84.

15. Heavner JE, Kaye AD, Lin BK, King T. Recovery of elderly patients from two or more hours of desflurane or sevoflurane anaesthesia.
Br J Anaesth 2003; 91: 502-6.

16. Magni G, Rosa IL, Melillo G, Savio A, Rosa G. A comparison between sevoflurane and desflurane anesthesia in patients undergoing craniotomy for supratentorial intracranial surgery. Anesth Analg 2009; 109: 567-71.

17. White PF, Tang J, Wender RH, Yumul R, Stokes OJ, Sloninsky A, et al. Desflurane versus sevoflurane for maintenance of outpatient anesthesia: the effect on early versus late recovery and perioperative coughing. Anesth Analg 2009; 109: 387-93.

18. Kaskinoro K, Maksimow A, Långsjö J, Aantaa R, Jääskeläinen S, Kaisti K, et al. Wide inter-individual variability of bispectral index and spectral entropy at loss of consciousness during increasing concentrations of dexmedetomidine, propofol, and sevoflurane. $\mathrm{Br}$ J Anaesth 2011; 107: 573-80. 